\title{
An advanced empirical model for quantifying the impact of heat and climate change on human physical work capacity
}

\author{
Josh Foster ${ }^{1}$ - James W. Smallcombe ${ }^{1} \cdot$ Simon Hodder ${ }^{1} \cdot$ Ollie Jay $^{2} \cdot$ Andreas D. Flouris $^{3} \cdot$ Lars Nybo $^{4}$. \\ George Havenith ${ }^{1}$
}

Received: 5 November 2020 / Revised: 14 January 2021 / Accepted: 23 February 2021 / Published online: 5 March 2021

(C) The Author(s) 2021

\begin{abstract}
Occupational heat stress directly hampers physical work capacity (PWC), with large economic consequences for industries and regions vulnerable to global warming. Accurately quantifying PWC is essential for forecasting impacts of different climate change scenarios, but the current state of knowledge is limited, leading to potential underestimations in mild heat, and overestimations in extreme heat. We therefore developed advanced empirical equations for PWC based on 338 work sessions in climatic chambers (low air movement, no solar radiation) spanning mild to extreme heat stress. Equations for PWC are available based on air temperature and humidity, for a suite of heat stress assessment metrics, and mean skin temperature. Our models are highly sensitive to mild heat and to our knowledge are the first to include empirical data across the full range of warm and hot environments possible with future climate change across the world. Using wet bulb globe temperature (WBGT) as an example, we noted $10 \%$ reductions in PWC at mild heat stress $\left(\mathrm{WBGT}=18^{\circ} \mathrm{C}\right.$ ) and reductions of $78 \%$ in the most extreme conditions $\left(\mathrm{WBGT}=40^{\circ} \mathrm{C}\right)$. Of the different heat stress indices available, the heat index was the best predictor of group level PWC $\left(R^{2}=\right.$ $0.96)$ but can only be applied in shaded conditions. The skin temperature, but not internal/core temperature, was a strong predictor of PWC $\left(R^{2}=0.88\right)$, thermal sensation $\left(R^{2}=0.84\right)$, and thermal comfort $\left(R^{2}=0.73\right)$. The models presented apply to occupational workloads and can be used in climate projection models to predict economic and social consequences of climate change.
\end{abstract}

\section{Introduction}

Human exposure to increased environmental heat directly impacts the global economy by decreasing occupational productivity during work hours (Flouris et al. 2018; Hsiang et al. 2017; Kjellstrom et al. 2018). The impact of hot weather on worker productivity was estimated to cost Australia EUR 5.52 billion per year (Zander et al. 2015), and in Germany, heat-

George Havenith

G.Havenith@lboro.ac.uk

1 Environmental Ergonomics Research Centre, School of Design and Creative Arts Loughborough University, Loughborough, Leicestershire LE11 3TU, UK

2 Thermal Ergonomics Laboratory, Faculty of Medicine and Health, The University of Sydney, Sydney, NSW, Australia

3 FAME Laboratory, University of Thessaly, Trikala, Greece

4 Department of Nutrition, Exercise and Sports, University of Copenhagen, Copenhagen, Denmark related productivity losses in 2004 were projected to cost between EUR 686.64 million and EUR 3.02 billion (Hübler et al. 2008). Occupational heat stress already has daily negative health and productivity impacts in many parts of the world and is therefore not precipitated by heat waves per se (Flouris et al. 2018; Kjellstrom et al. 2018). To correctly quantify the impact of environmental heat (mild to extreme) on human physical work capacity (PWC), accurate equations are required, which relate PWC to a wide range of thermal conditions. We describe below why existing models (Dunne et al. 2013; Kjellstrom et al. 2018; Zivin and Neidell 2014) presently used to inform those predictions have limited applicability, especially for use on a global scale. These considerations justify the development of a new series of empirically derived equations intended to quantify the loss in PWC more precisely across a wide range of environmental conditions. Such empirical equations have immediate application for those striving to evaluate the productivity and, thus, economic consequences of hot weather, particularly in different climate change scenarios arising from variations in projected greenhouse gas emissions. 


\section{Previous models: a critical analysis}

Efforts to model the loss in PWC with increasing heat have taken three distinct forms (Dunne et al. 2013; Kjellstrom et al. 2018; Zivin and Neidell 2014). In the first, with an absence of empirical data, Dunne et al. (2013) generated functions based on the American Conference of Governmental Industrial Hygienists (ACGIH)-recommended work/rest ratios for a given wet bulb globe temperature (WBGT), designed to limit core temperature from exceeding $38.0^{\circ} \mathrm{C}$. Those functions are highly conservative because their objective is to minimise the risk of core body temperature of the average worker exceeding $38.0^{\circ} \mathrm{C}$ (which would prevent workers at the top end of the body temperature distribution to exceed $41^{\circ} \mathrm{C}$ with risk of heat illness) (Malchaire et al. 2001). Second, the ACGIH recommendations were never intended to represent the decline in labour output and are unlikely to accurately predict output from self-paced work in a large and diverse working population. Thus, while ACGIH recommendations show that continuous work is safe at $26^{\circ} \mathrm{C}$ WBGT, and therefore indicate no productivity loss in Dunne et al.'s (2013) model, physiological studies indicate that work output is reduced in this scenario, due to elevations in cardiovascular strain (Galloway and Maughan 1997).

Second, Kjellstrom et al. (2018) modelled field data from agriculture (Sahu et al. 2013) and gold mining (Wyndham 1969) and demonstrate a nonlinear (sigmoidal) decrease in PWC as a function of WBGT. Those data represent an improvement in ecological validity because the source data are based on scenarios where workers could freely adjust their pace. However, the source data (Sahu et al. 2013; Wyndham 1969) are highly context specific and, as such, are of limited utility on a global scale. Firstly, the reference condition to which subsequent hot trials were compared was already warm, i.e. $27^{\circ} \mathrm{C}$ WBGT, which far exceeds optimal thermal conditions for human performance and thus greatly affects the sensitivity of the model at modest levels of heat. Secondly, the model is based only on well-conditioned, highly heat acclimatised, and incentivised workers, which represents the upper limit of PWC in relation to each climate, but will over-predict PWC in less fit, unacclimatised workers. The latter is relevant for those who reside in climates that experience more transient or unaccustomed peaks in temperature for which they are not physically prepared. To quote Cyril Wyndham, the primary source for the data used by Kjellstrom et al. (2018), "these curves apply only to men in the high state of acclimatization of the Bantu in the gold mines in South Africa and to men carrying out physical work at a moderate rate under direct supervision" (Wyndham 1969). Finally, the PWC predictions from Dunne et al. (2013) and Kjellstrom et al. (2018) can only be adjusted based on WBGT, which limits their utility if an alternative heat stress metric (i.e. universal thermal climate index (UTCI), wet bulb, heat index, humidex) is preferred. A global model should have the capacity to be adjusted to incorporate any heat stress assessment metric, of which many are currently adopted for different applications (Havenith and Fiala 2015).

Finally, Zivin and Neidell (2014) model the impact of hot weather on the decrease in time allocated to labour per day, based on data from the American Time Use Survey (ATUS) (Hamermesh et al. 2005) combined with local weather station data. Hsiang et al. (2017) use the model to evaluate the contribution of labour loss to economic impacts of climate change in the USA. For industries considered vulnerable to heat, the model demonstrates reductions of up to 1 -h lost labour time per day time when air temperature reaches $38^{\circ} \mathrm{C}$. Importantly however, the model does not address the heat-induced reduction in labour effort during work (presenteeism) and is apparently insensitive to any change in humidity, a key determinant of the overall heat stress intensity (Malchaire et al. 2001; Maughan et al. 2012; Raymond et al. 2020). Field studies demonstrate that functional/quality labour time is very sensitive to heat, with total output during work declining with increasing heat (Ioannou et al. 2017; Kalkowsky and Kampmann 2006; Sahu et al. 2013). Furthermore, presenteeism formed a major contribution to heat-induced reductions in economic output in Australia (Zander et al. 2015).

The aim of this study was to produce an advanced, novel, empirical model for the loss in PWC with environmental heat stress. We aim to generate models of PWC based off a suite of heat stress assessment metrics, air temperature and humidity, and body temperatures. We intend the models to be used primarily for projecting the impact of heat waves and climate change on human physical work capacity.

\section{Materials and Methods}

\section{Location and timeline}

The data collection took place in custom-made environmental chambers located within the Environmental Ergonomics Research Centre, Loughborough University. Data collection began in July 2017 and terminated in October 2019.

\section{Participants}

Young adult males, primarily from a student population, were recruited for this study. The total number of trials completed by each participant $(n=40)$ varied (average $=12$, range $=4$ to 35). Participant characteristics are shown for each experimental group in Table 1. 
Table 1 Participant

characteristics. Data are presented as means \pm standard deviation. The data range is presented in parentheses.

\begin{tabular}{|c|c|c|c|c|}
\hline \multirow{2}{*}{$\begin{array}{l}\text { Variable } \\
\text { Age (years) }\end{array}$} & \multicolumn{2}{|c|}{ Low-clothing coverage $(n=20)$} & \multicolumn{2}{|c|}{$\begin{array}{l}\text { High-clothing } \\
\text { coverage }(n=20)\end{array}$} \\
\hline & $25 \pm 3$ & $(20-29)$ & $24 \pm 2$ & $(20-28)$ \\
\hline Height $(\mathrm{cm})$ & $178 \pm 5$ & $(170-190)$ & $180 \pm 6$ & $(172-192)$ \\
\hline Mass (kg) & $77 \pm 11$ & $(61-101)$ & $79 \pm 11$ & $(62-99)$ \\
\hline Body surface area $\left(\mathrm{m}^{-2}\right)$ & $1.9 \pm 0.2$ & $(1.7-2.3)$ & $2.0 \pm 0.2$ & $(1.7-2.3)$ \\
\hline Body mass index $\left(\mathrm{kg} \cdot \mathrm{m}^{-2}\right)$ & $24 \pm 2$ & $(21-29)$ & $24 \pm 2$ & $(21-29)$ \\
\hline Body fat (\%) & $18 \pm 5$ & $(11-26)$ & $15 \pm 5$ & $(8-26)$ \\
\hline$\dot{V} \mathrm{O}_{2 \max }\left(\mathrm{L} \cdot \min ^{-1}\right)$ & $3.8 \pm 0.8$ & $(2.7-6.2)$ & $4.0 \pm 0.7$ & $(2.7-5.3)$ \\
\hline$\dot{V} \mathrm{O}_{2 \max }\left(\mathrm{mL} \cdot \mathrm{kg}^{-1} \cdot \mathrm{min}^{-1}\right)$ & $50 \pm 9$ & $(40-67)$ & $51 \pm 8$ & $(39-64)$ \\
\hline
\end{tabular}

$\dot{V} O_{2 \max }$ maximal oxygen consumption, $L$ litres, $m L$ millilitres

\section{A fixed cardiovascular strain protocol to model self- pacing}

In the present study, we define PWC as 'the maximum physical work output that can be reasonably expected from an individual performing moderate to heavy work over an entire shift'. To more effectively investigate how heat stress impacts PWC, we designed a laboratory protocol to simulate these pacing behaviours, capturing the reduction in performance in warming climates, relative to a cool reference climate of $15^{\circ} \mathrm{C}$. This air temperature was chosen to limit any effect of temperature on heart rate without causing substantial cold stress. Air temperatures as low as $20^{\circ} \mathrm{C}$ have been shown to decrease PWC relative to a cooler climate (Galloway and Maughan 1997), and $15^{\circ} \mathrm{C}$ has been used previously to determine the impact of heat stress on human physical performance (Marino et al. 2000). The protocol aimed at measuring the amount of work the body can generate at a fixed, maximally acceptable cardiovascular strain $\left(130 \mathrm{~b} \cdot \mathrm{min}^{-1}\right)$ across a broad spectrum of air temperature $\left(T_{\mathrm{a}}, 25-50^{\circ} \mathrm{C}\right)$ and relative humidity $(20$ $80 \%$ ). The spectrum of environmental conditions represents mild exposures to more extreme levels that extend into future worst-case greenhouse gas emission scenarios (i.e. WBGT $18-40^{\circ} \mathrm{C}$ ) (Kjellstrom et al. 2018; Pal and Eltahir 2016). The experiments were up to 1 -h duration and took place within an environmental chamber, where participants walked on a treadmill that automatically adjusted its speed and incline to maintain a stable heart rate of $130 \mathrm{~b} \cdot \mathrm{min}^{-1}$. A large body of evidence from the field demonstrates that workers pace themselves based on their heart rate/perceived exertion, resulting in similar values for working heart rate independent of the climate (Kalkowsky and Kampmann 2006; Mairiaux and Malchaire 1985; Miller et al. 2011; Vogt et al. 1983; Wyndham 1969), but at the cost of productivity (Kjellstrom et al. 2018). Recently, this approach was employed successfully to investigate the effect of electric fans on work output in mild heat (Jay et al. 2019).

The fixed cardiovascular strain protocol is highly sensitive to changes in PWC with heat because as the environmental heat load increases, a growing proportion of the $130 \mathrm{~b} \cdot \mathrm{min}^{-1}$ heart rate is used to ensure delivery of warm blood to the skin for heat loss (Rowell 1974). Consequently, a lower proportion of the cardiac output (blood leaving the heart per minute) can be used to fuel muscular work during heat stress for a fixed heart rate, resulting in a reduced total work output (Rowell 1974). The fixed heart rate protocol likely minimises any impact of the environment on stroke volume, since any reduction in stroke volume during heat stress is related to the increase in heart rate (due to impacts on ventricular filling time) (Chou et al. 2019). The target working heart rate was chosen based on three key sources. Firstly, the World Health Organisation classification of relative work intensities indicates that $130 \mathrm{~b}$. $\mathrm{min}^{-1}$ during the work periods represents the demarcation between moderate and heavy strain (Andersen 1978), considered to be the maximal acceptable workload for sustained work periods (Bernard and Kenney 1994). Secondly, field observations indicated that physical work in the heat was regulated at a working heart rate close to $130 \mathrm{~b} \cdot \mathrm{min}^{-1}$ in miners (Wyndham 1973) and in glass furnace workers (Mairiaux and Malchaire 1985), regardless of the heat load of the environment. Thirdly, during a working period of four work bouts with different heat loads, absolute heart rate was on average $130 \mathrm{~b} \cdot \mathrm{min}^{-1}$ at the end of each self-paced work bout (Vogt et al. 1983). The field data indicate that $130 \mathrm{~b} \cdot \mathrm{min}^{-1}$ is an acceptable upper level of physiological strain for the maintenance of physical work in thermally challenging environments, at the cost of reduced muscular work as the heat stress increases.

\section{Limiting the development of physiological heat adaptation}

To limit the development of physiological heat adaptation, the number of experiments was set to a maximum of three per week, but typically did not exceed two per week. Given that sustained elevations in core body temperature and skin temperature are required to elicit heat adaptation (Chen and Elizondo 1974) and that the fixed heart rate approach 
minimises elevations in core temperature (Fig. 5c), no relevant level of heat adaptation due to the testing is assumed. Moreover, the fixed heart rate method employed limits any potential effect of subjective adaptation to frequent heat exposure. Similarly, no impact of testing over a long period with different seasons is expected in the present experiment as prior research shows no significant seasonal effect on thermal or cardiovascular responses to work in the heat (Bain and Jay 2011), reducing the possibility of heat adaptation that could have impacted the results. However, since some participants completed many trials, we cannot completely dismiss the influence of mild heat adaptation over such a long experimental period. Due to potential thermoregulatory adaptations, individuals were not permitted to take part in any experimental procedures if they were heat acclimated or acclimatised (Garrett et al. 2011), e.g. by spending time in very hot climates or by participating in acclimatisation experiments.

\section{Experimental controls}

Individual participants completed experimental sessions at the same time of day to minimise the effect of circadian rhythm on outcome variables (Waterhouse et al. 2004). Participants were asked to arrive hydrated before commencement of laboratory testing sessions and to refrain from caffeine on the day of each trial and alcohol and vigorous exercise $24 \mathrm{~h}$ prior to each trial.

\section{Anthropometry and submaximal exercise/fitness test (Visit 1)}

Anthropometry was conducted with participants wearing shorts, T-shirt, and socks. Body mass was measured to the nearest gram using a high-precision digital scale (Metter Toledo kcc150, Metter Toledo, Leicester, UK), and stature was measured to the nearest $0.01 \mathrm{~m}$ using a wall-mounted stadiometer (Holtain, Crosswell, UK). Body composition was determined by bioelectrical impedance (Tanita MC780MA, TANITA Corporation, Tokyo, Japan).

A gradient-based incremental submaximal exercise test was conducted in an environmental chamber regulated at $18^{\circ} \mathrm{C}, 40 \%$ rh and was performed on a treadmill (Mercury Medical, h/p/cosmos sports \& medical Gmbh, Germany). This test consisted of a maximum of six, 3-min stages. During the test, the treadmill speed was fixed at $4.5 \mathrm{~km} \cdot \mathrm{h}^{-1}$, and the gradient was increased by $5 \%$ every 3 min until a steady-state heart rate of $85 \%$ age-predicted maximum was elicited. Expired air and heart rate were continuously monitored using an online gas analysis system (Quark CPET, COSMED, Albano Laziale, Rome) and short-range telemetry (Polar PE4000, Polar Electro, Kempele, Finland), respectively. The oxygen uptake and heart rate data collected during the submaximal treadmill test were extrapolated to estimate maximal oxygen consumption $\left(\dot{V} \mathrm{O}_{2 \max }\right)$ (American College of Sports Medicine 2013).

\section{Experimental trials}

Upon arrival, participants inserted a rectal thermistor (VIAMED, Yorkshire, UK) to a depth of $10 \mathrm{~cm}$ past the anal sphincter. They then provided a urine sample for assessment of urine specific gravity. To ensure a state of euhydration, if urine specific gravity was $>1.020$, participants were asked to drink $\sim 500 \mathrm{~mL}$ water and to provide a second urine sample after $20 \mathrm{~min}$ (Armstrong et al. 1994). To monitor skin temperature $\left(T_{\text {skin }}\right)$, skin thermistors were placed on the belly of the pectoralis major $\left(T_{\text {chest }}\right)$, triceps $\left(T_{\text {arm }}\right)$, rectus femoris $\left(T_{\text {thigh }}\right)$, and gastrocnemius $\left(T_{\text {calf }}\right)$. The mean $T_{\text {skin }}$ was then calculated based on the equation provided by Ramanathan (Ramanathan 1964). The value for $T_{\text {skin }}$ was reported as the average score during a hot work trial.

$$
T_{\text {skin }}=0.3\left(T_{\text {chest }}+T_{\text {arm }}\right)+0.2\left(T_{\text {thigh }}+T_{\text {calf }}\right)\left[{ }^{\circ} \mathbf{C}\right]
$$

Participants entered the environmental chamber wearing one of two ensembles. In the low-clothing coverage trials, participants donned underwear, standardised shorts, socks, and trainers. In the high-clothing coverage trials, the participants donned underwear, a standardised cotton t-shirt, and an appropriately sized, standardised full body protective coverall (65\% polyester, $35 \%$ cotton). The intrinsic clothing insulations of the low- and high-clothing coverage ensembles were estimated as 0.04 and $0.133 \mathrm{~m}^{-2} \cdot \mathrm{K} \cdot \mathrm{W}^{-1}(0.26$ and 0.86 Clo), respectively, based on the reference tables provided in the international standard (ISO9920 2009). Using Eq. 31 in the standard, the evaporative resistance can be estimated as 0.007 and $0.024 \mathrm{~m}^{-2} \cdot \mathrm{kPa} \cdot \mathrm{W}^{-1}$ for the low- and high-clothing coverage ensembles, respectively.

Various data acquisition systems were used to log skin and core temperature (Grant Squirrel SQ2020, Grant Instruments Ltd., Corby, UK), WBGT (Quest temp model 34), air temperature, relative humidity, and air velocity (Testo Ltd, model 435-2 Alton, Hampshire, UK) at 1-min intervals. Ratings of thermal comfort, rating of perceived exertion (RPE), and thermal sensation were taken every $5 \mathrm{~min}$ and reported as median of the work trial. Images of the scales used are shown in the online supplement (Figure S4). 'The descriptors for each of the scales comply with international standards for measuring thermal comfort and sensation' (ISO10551 2001). The comfort and sensation scales were modified to include intermediate numeric values to provide more choice to the participants regarding their precise comfort and thermal sensation level. The term 'extremely uncomfortable' was not included in our scale due to its limited practical use in our work.

Participants were removed from the climate chamber and the trial terminated if core temperature reached $39^{\circ} \mathrm{C}$. 


\section{Physical work simulation}

One hour of treadmill-based walking was then commenced adhering to the following protocol. The treadmill was programmed to control workload to achieve the desired heart rate of $130 \mathrm{~b} \cdot \mathrm{min}^{-1}$. With this setting applied, the treadmill automatically manipulated the speed and grade to ensure heart rate was maintained at the predefined target. The treadmill speed and grade were never manually controlled by the researchers or participants. The treadmill elevation remained at $0 \%$ until the speed reached the threshold $6 \mathrm{~km} \cdot \mathrm{h}^{-1}$; thereafter, the treadmill regulated the elevation based on the difference between the actual and desired heart rate. The maximum test duration was set at $1 \mathrm{~h}$, but exercise ceased if the treadmill speed reached zero, i.e. the participant having a heart rate of $130 \mathrm{~b}$. $\mathrm{min}^{-1}$ at rest. In this scenario, participants were removed from the chamber and the trial did not continue.

\section{Calculation of percentage physical work capacity}

The 'minimum mechanics' model was used to predict walking metabolism (kJ) (Ludlow and Weyand 2017). This equation was preferred over the widely used American College of Sports Medicine (ACSM) and Pandolf equations (Pandolf et al. 1977) due to its consistently stronger predictive accuracy over a wide range of speeds and grades (Ludlow and Weyand 2017). PWC was based on the total energy generated/ expenditure (EE) above resting in each trial relative to that achieved in a cool reference condition, expressed as a percentage. The rate of work EE in kilojoules per minute $\left(\mathrm{kJ} \cdot \mathrm{min}^{-1}\right)$ during each minute of work was calculated as below in Eq. 1, with the cumulative total used as final work EE (in $\mathrm{kJ}$ ):

$$
\begin{gathered}
\text { Work } E E=\sum_{t=1}^{60}\left[0.32 \cdot G(t)+3.28+(1+0.19 \cdot G(t)) \cdot\left(2.66 \cdot v(t)^{2}\right)\right] \\
\cdot\left(19.61+\frac{R Q(t)-0.707}{0.293} \cdot 1.51\right)[k J]
\end{gathered}
$$

where $G(t)$ is the slope of the treadmill expressed in percent grade at time $t, v(\mathrm{t})$ is velocity of walking expressed in meters per second, and $\mathrm{RQ}(\mathrm{t})$ is the respiratory quotient that was assumed to be 0.85 (Cramer and Jay 2019). The summation function $\left(\sum\right)$ denotes that the output of the equation is summed every $1 \mathrm{~min}(t=1)$ until a stopping point of $60 \mathrm{~min}$, accounting for the change in each variable over time. Part 1 of the equation (Ludlow and Weyand 2017) calculates the net volume of oxygen consumed $\left(\dot{V} \mathrm{O}_{2-\text { net }}\right.$, in $\mathrm{mL} \cdot \mathrm{kg}$ body mass ${ }^{-1}$. $\mathrm{min}^{-1}$ ) to fuel exercise, i.e. not including resting $\dot{V O}_{2}$. Part 2 of the equation converts the former into $\mathrm{kJ} \cdot \mathrm{min}^{-1}$. The cumulative $\mathrm{EE}$ for each trial was used to calculate total $\mathrm{EE}$ (in $\mathrm{kJ}$ ) in each individual trial. The validity of the prediction equation for EE was tested against 365 expired air samples (5-min average) from a metabolic cart (Quark CPET, COSMED, Albano Laziale, Rome). Expired air measurements were taken at 3 time points (5-10, 30-35, and 50-55 $\mathrm{min}$ ), and the $\mathrm{kJ}$. $\min ^{-1}$ subsequently compared with the prediction equation (Ludlow and Weyand 2017). See supplementary file for correlations and Bland-Altman analysis (Figure S5 and S6).

Percentage PWC in each hot trial was determined by expressing the total energy generated from metabolic processes (above resting) in the hour of work relative to that achieved in a reference cool condition (Eq. 2)

Physical Work Capacity $=\left(\frac{\operatorname{Hot}_{k J}}{\operatorname{Cool}_{k J}}\right) \times 100$

where Cool $_{\mathrm{kJ}}$ is the total energy generated (kilojoules, $\mathrm{kJ}$ ) above resting metabolism in the cool reference condition and $H_{o t} t_{\mathrm{kJ}}$ is the total energy generated above resting metabolism in each heat stress trial. Empirical models for the decrease in PWC under heat stress were then generated using data from 338 trials separated into low (181trials) and high (157 trials) clothing coverage. Figure 1 demonstrates how environmental heat stress changes skin temperature and consequently treadmill (physical work) parameters for a representative participant.

Figure 2 illustrates the two clothing ensembles worn in the low- and high-clothing coverage conditions. All trials were conducted in a still environment $\left(\sim 0.2 \mathrm{~m} \cdot \mathrm{s}^{-1}\right)$, and scores for PWC used in the model were taken as the average for a group of participants in each climate condition. Low- and highclothing coverage trials were completed in 22 climatic conditions, with an average of 7 trials per condition. The performance results for individual climates are presented in Table 2 .

\section{Calculation of heat stress indices}

\section{Wet bulb globe temperature}

The WBGT was measured empirically using a WBGT monitor (Quest temp model 34). The value used in the model was the average over the course of each work bout.

\section{Aspirated (psychrometric) wet bulb temperature}

Aspirated wet bulb temperature was calculated based on the formula provided by Bernard and Pourmoghani (1999):

$T_{w b}=0.376+5.79 P_{\mathrm{a}}+\left(0.388-0.0465 \mathrm{P}_{a}\right) T_{\mathrm{db}} \quad\left[{ }^{\circ} \mathrm{C}\right]$

where $P_{\mathrm{a}}$ is the ambient water vapour pressure measured in $\mathrm{kPa}$ and $T_{\mathrm{db}}$ is the dry bulb temperature (air temperature). $P_{\mathrm{a}}$ was calculated by (Parsons 2010):

$P_{\mathrm{a}}=\left[e^{\left(18.956-\frac{4030.18}{T_{\mathrm{a}}+235}\right)}\right] \times \frac{R h}{100} \quad[\mathrm{kPa}]$

where $T_{\mathrm{a}}$ is the ambient temperature in ${ }^{\circ} \mathrm{C}$ and $\mathrm{RH}$ is the relative humidity $(0-100)$. 


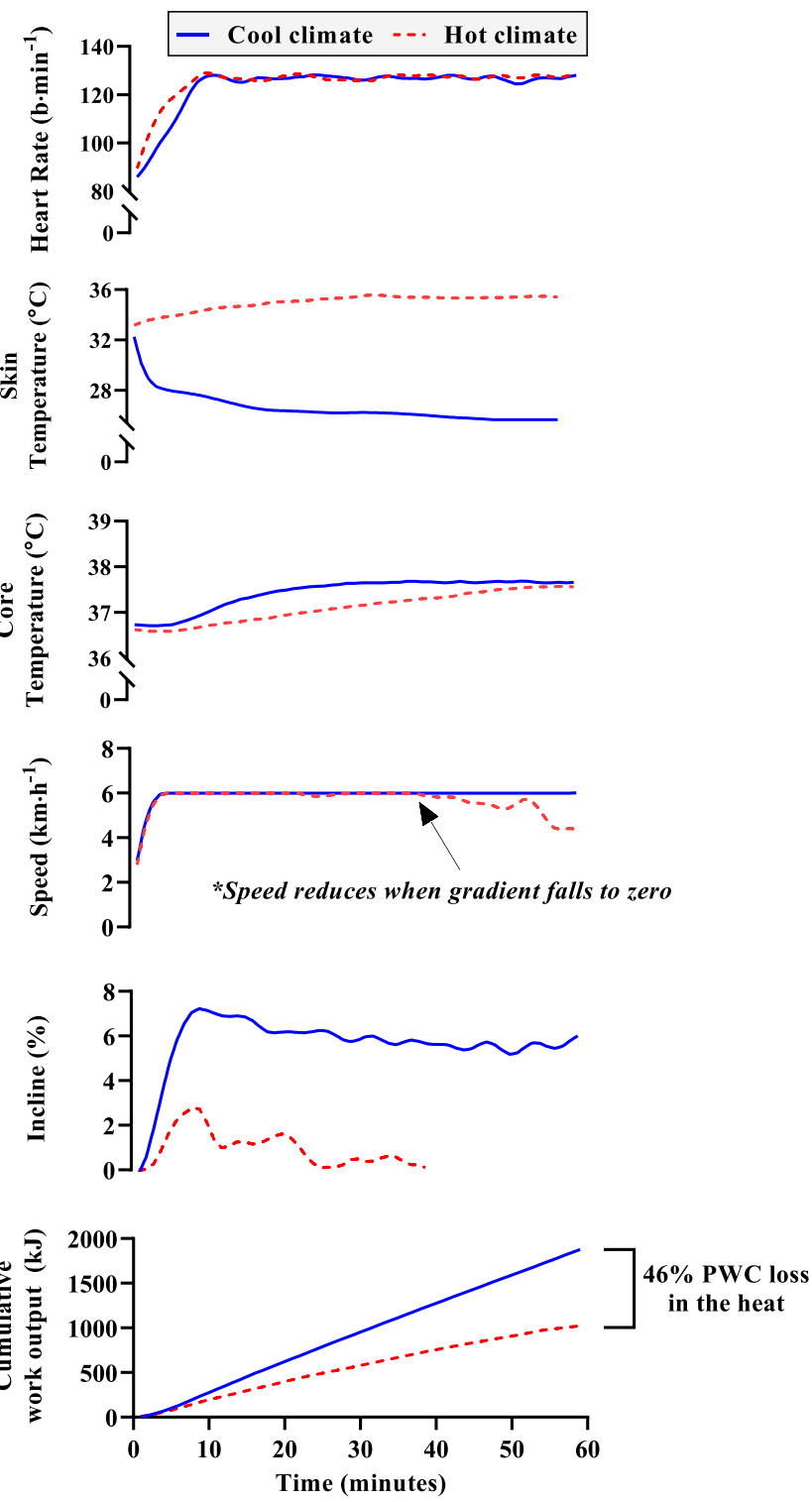

Fig. 1 Fixed heart rate protocol measures how climatic heat impacts work capacity. Field studies demonstrate that individuals work at a similar heart rate despite changes in climatic heat due to adjustments in pacing. Although the heart rate profile is not different between conditions, heat stress causes a substantial increase in skin temperature, causing dramatic reductions in overall energy generated. Core (rectal) temperature responses in the early phase of the exposures are driven primarily by metabolic heat production (work rate). Changes to cumulative energy expenditure were used to calculate physical work capacity (PWC). Example data is shown for a representative participant for the reference condition $\left(15^{\circ} \mathrm{C}, 50 \%\right.$ humidity, solid blue line $)$ and a hot condition $\left(40^{\circ} \mathrm{C}, 20 \%\right.$ humidity, dashed red line)

\section{Universal thermal climate index}

The UTCI was determined using an excel calculator (www. climatechip.org/excel-wbgt-calculator), using the regression polynomial provided by the operational procedure of UTCI
(Bröde et al. 2012). The input values used for the calculation were $T_{\mathrm{a}}, \mathrm{RH}$, globe temperature, and air velocity. The UTCI macro was converted into VBA from the FORTRAN source code supplied at the UTCI site (www.utci.org/utci_doku.php).

\section{Humidex}

The humidex was calculated based on Masterton and Richardson (1979) and Rana et al. (2013):

$$
\text { Humidex }=T_{\mathrm{a}}+\frac{5}{9}\left(\left[6.112 \times 10^{\left(\frac{7.5 T_{\mathrm{a}}}{237.7+T_{\mathrm{a}}}\right)} \times \frac{R h}{100}\right]-10\right)\left[{ }^{\circ} \mathrm{C}\right]
$$

\section{Heat index}

The heat index was calculated based on Rothfusz (1990):

$$
\begin{aligned}
\text { Heat Index } & =-42.379+2.04901523 T_{a}+10.14333127 R h \\
& -0.22475541 T_{a} \cdot R h-6.83783 \times 10^{-3} T_{a}^{2}-5.481717 \\
& \times 10^{-2} R h^{2}+1.22874 \times 10^{-3} T_{a}^{2} \cdot R h+8.5282 \\
& \times 10^{-4} T_{a} \cdot R h^{2}-1.99 \times 10^{-6} T_{a}^{2} \cdot R h^{2}\left[{ }^{\circ} \mathrm{F}\right]
\end{aligned}
$$

where $T_{\mathrm{a}}$ is in degrees Fahrenheit and RH is $0-100$. The heat index in Fahrenheit $\left(H I_{\mathrm{F}}\right)$ was converted to degrees Celsius by:

$$
\text { Heat Index }=\left(H I_{\mathrm{F}}-32\right) \times \frac{5}{9} \quad\left[{ }^{\circ} \mathrm{C}\right]
$$

\section{Statistical analysis}

All statistical models using heat stress indices were generated using GraphPad Prism version 8. Models incorporating air temperature and relative humidity were generated using nonlinear equation builder in IBM SPSS Statistics version 25 . The reduction in PWC caused by heat was modelled using the collected data in relation to (i) commonly used heat stress indices and (ii) air temperature and humidity. Heat stress indices included in the analysis are the WBGT, aspirated (psychrometric) wet bulb temperature $\left(T_{\mathrm{wb}}\right)$, universal thermal climate index (UTCI), humidex, and heat index (see methodology for calculations). Additional formulas are available in the online supplement if using apparent temperature, standard effective temperature, the Oxford index, perceived temperature, physiological equivalent temperature, and the modified physiological equivalent temperature (Tables S4, S5, and S6). Each index is used as a general climate strain index, and we acknowledge in Table 3 that some indices (i.e. heat index) do not account for solar radiation and wind speed in their calculation. Such parameters are being considered in ongoing 


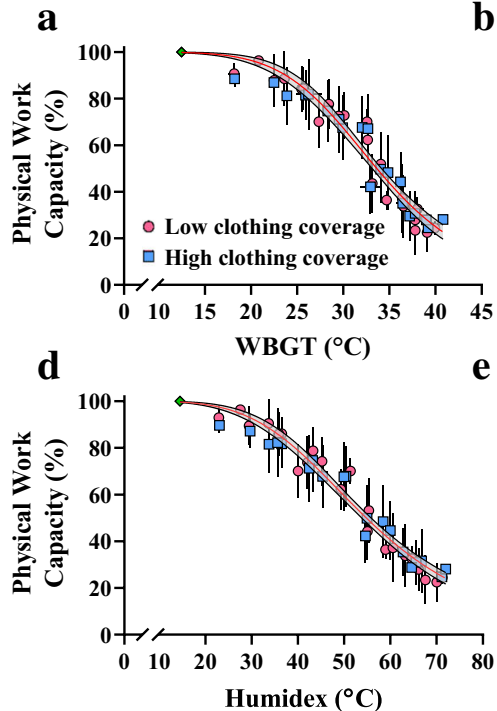

Fig. 2 Models for the reduction in physical work capacity during heat stress. Models are presented against five heat stress indices (a-e) and for air temperature and relative humidity (f). The data used to form projections are taken as the average physical work capacity from each air temperature and humidity combination, pooling that of low- (pink

works from our lab in which we assess their independent effects on PWC across the heat stress spectrum (Foster et al. 2020b; Smallcombe et al. 2019b). These indices were chosen due to their frequent application in occupational hygiene, climate modelling, biometeorology, and weather reports.

In line with the approach of Kjellstrom et al. (2018), we considered a sigmoidal model to be the most appropriate fit to our data. A sigmoidal model allows for logical upper and lower limits of 100 and $0 \%$ PWC, respectively, which can be enclosed in one equation without adding limiters.

The data was modelled according to the following formula in Eq. 8:

Physical Work Capacity\% $=\frac{100}{1+\left(\frac{P W C 50}{x}\right)^{\text {HillSlope }}}$

where PWC50 is the value of $x$ that elicits 50\% PWC and Hillslope defines the steepness of the curve. The function has a 'top' and 'bottom' plateau of 100 and $0 \%$, respectively, but we stress that our models are only validated within the span of conditions tested (see Table 2), where PWC reached a minimum of $20 \%$ in our cohort of participants. In GraphPad Prism, the 'top' and 'bottom' parameters were fixed at 100 and 0 , respectively. The HillSlope and PWC50 parameters were calculated from the software to find the optimal fit to the data (producing the least variance).

The model equation template was adapted when incorporating $T_{\mathrm{a}}$ and humidity only, instead of using a heat stress index. Therefore, if using $T_{\mathrm{a}}$ as the value of $x$, the values of $P W C 50$ and Hillslope are replaced with functions/models that represent their change as a function of relative humidity (Eq. c

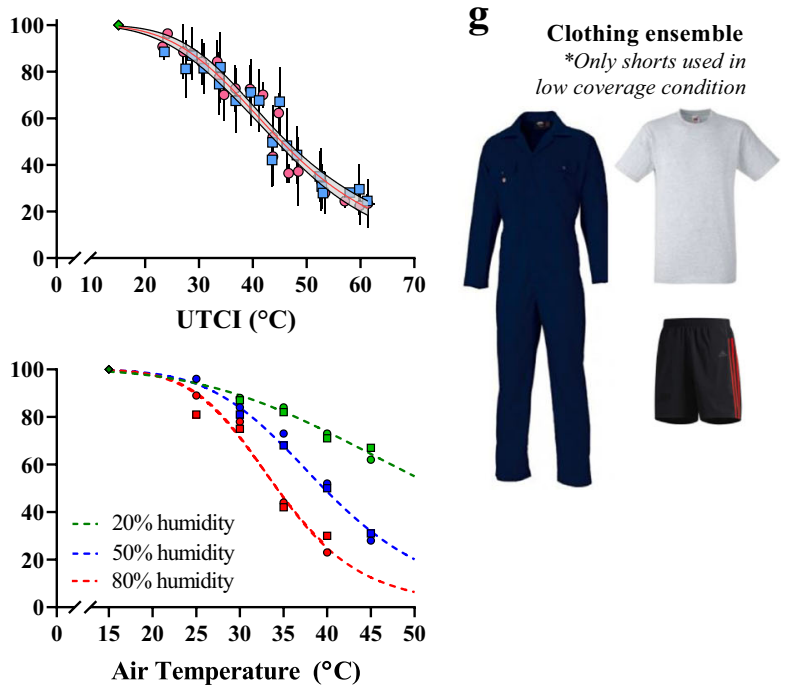

circles) and high- (blue squares) clothing coverage trials (displayed in panel $g$ ). Model $f$ was the highest performing overall, but model $e$ (heat index) was the highest performing among different heat stress indices. Model analytics are available in Table 3

9):

\section{Physical Work Capacity\%}

$$
=\frac{100}{1+\left[\frac{(a \cdot \operatorname{Ln}(R H)+b)}{T_{a}}\right]^{[c \cdot \operatorname{Ln}(R H)+d]}}
$$

where $R H$ is the relative humidity (data valid from 20 to $80 \%$ ) and $\operatorname{Ln}(R H)$ denotes a transformation of $R H$ to the natural logarithm; the $a, b, c$, and $d$ parameters are empirically derived constants.

\section{Parameter tuning}

The models that replace 'PWC50' and 'Hillslope' were derived by the following methodology.

1. For three different relative humidity levels $(20,50$, and $80 \%$ ), independent sigmoidal models were produced, which describe the loss in PWC as a function of air temperature (Fig. 2f). All three humidity models produce different values for ' $P W C 50$ ' and 'Hillslope' to fit the data.

2. Basic linear models were then produced, which show how each of these parameters changes as a function of relative humidity. For example, relative humidity is the $x$ value, and PWC50 is the $y$ value in the equation. Repeating this step for Hillslope, two models have now been generated, which describe how PWC50 and Hillslope change as a function of relative humidity. 
Table 2 Percentage physical work capacity across a broad range of air temperature and relative humidity combinations with high- and lowclothing coverage

\begin{tabular}{|c|c|c|c|c|c|}
\hline \multicolumn{3}{|c|}{$\underline{\text { Low-clothing coverage }}$} & \multicolumn{3}{|c|}{ High-clothing coverage } \\
\hline$T_{\mathrm{a}}$ & RH & $\mathrm{PWC} \% \pm \mathrm{SD}$ & $T_{\mathrm{a}}$ & RH & $\mathrm{PWC} \% \pm \mathrm{SD}$ \\
\hline \multirow[t]{4}{*}{25} & & & 25 & & \\
\hline & 20 & $91 \pm 4$ & & 20 & $88 \pm 3$ \\
\hline & 50 & $96 \pm 1$ & & ----- & ------- \\
\hline & 80 & $89 \pm 11$ & & 80 & $81 \pm 12$ \\
\hline \multirow[t]{4}{*}{30} & & & 30 & & \\
\hline & 20 & $88 \pm 11$ & & 20 & $87 \pm 7$ \\
\hline & 50 & $84 \pm 10$ & & 50 & $81 \pm 11$ \\
\hline & 80 & $77 \pm 11$ & & 80 & $75 \pm 13$ \\
\hline \multirow[t]{5}{*}{35} & & & 35 & & \\
\hline & 20 & $84 \pm 9$ & & 20 & $82 \pm 15$ \\
\hline & 35 & $70 \pm 11$ & & ---- & -------- \\
\hline & 50 & $73 \pm 10$ & & 50 & $68 \pm 14$ \\
\hline & 80 & $44 \pm 12$ & & 80 & $42 \pm 11$ \\
\hline \multirow[t]{7}{*}{40} & & & 40 & & \\
\hline & 20 & $73 \pm 10$ & & 20 & $71 \pm 14$ \\
\hline & 40 & $70 \pm 5$ & & 40 & $68 \pm 13$ \\
\hline & 50 & $52 \pm 14$ & & 50 & $50 \pm 14$ \\
\hline & 60 & $36 \pm 4$ & & 60 & $48 \pm 16$ \\
\hline & 70 & $34 \pm 13$ & & 70 & $35 \pm 11$ \\
\hline & 80 & $23 \pm 10$ & & 80 & $30 \pm 11$ \\
\hline \multirow[t]{5}{*}{45} & & & 45 & & \\
\hline & 20 & $62 \pm 9$ & & 20 & $68 \pm 15$ \\
\hline & 40 & $37 \pm 15$ & & 40 & $45 \pm 13$ \\
\hline & 50 & $28 \pm 9$ & & 50 & $31 \pm 10$ \\
\hline & 60 & $22 \pm 8$ & & 60 & $25 \pm 3$ \\
\hline \multirow[t]{3}{*}{50} & & & 50 & & \\
\hline & 30 & $33 \pm 5$ & & 30 & $29 *$ \\
\hline & 40 & $24 \pm 3$ & & 40 & $28 *$ \\
\hline
\end{tabular}

$T_{a}$ air temperature, $R H$ relative humidity, $P W C \%$ percentage physical work capacity, $S D$ standard deviation

* No standard deviation reported because $n=1$

3. The model framework from Eq. 9 was input into SPSS nonlinear equation builder. The initial 'best guess' estimates for the coefficients $a, b, c$, and $d$ were derived from the models produced in step 2. Running the nonlinear equation in SPSS produces the model where PWC can be predicted from air temperature and relative humidity, as shown in Table 3.

\section{Results}

Table 2 shows the change in PWC in each environmental condition, based on either low- or high-clothing coverage.
Here, we show that PWC is strongly influenced by air temperature, humidity, and, to a mild extent in our study, the level of clothing coverage.

Since the clothing types tested only had a limited impact on PWC, models were generated from the pooled dataset, as shown in Fig. 2a-e and separated for clothing (Fig. 4). The equations are displayed in Table 3 for the pooled clothing dataset. Equations for the separate low- and high-clothing coverage conditions shown in Fig. 4 are available in the supplementary material (Tables S1 and S2). Table 3 also acknowledges which equations are likely to be accurate in outdoor working conditions, with the added burden of thermal radiation from the sun, which increases heat strain and reduced physical performance independently of $T_{\mathrm{a}}$ and humidity (Hodder and Parsons 2007; Otani et al. 2016). Figure 3 shows the output from the $T_{\mathrm{a}}$ and humidity model as a matrix. The green area shows minimal reduction in PWC regardless of the $T_{\mathrm{a}}$ and humidity combination. The humidity has an increasingly strong impact on $\mathrm{PWC}$ at $25^{\circ} \mathrm{C} T_{\mathrm{a}}$ and above. Figure 5 demonstrates the physiological manifestations underlying the observed reduction in PWC with heat. Whereas PWC was predicted strongly by the increase in skin temperature, rectal temperature and mean body temperature were not useful predictors. For those reasons, perceptual responses shown in Fig. 5 were expressed relative to the change in skin temperature only. Table 4 shows how PWC can be modelled based on skin temperature and how thermal sensation and thermal comfort can be predicted based on the skin temperature. Models are available in the supplementary material to predict skin temperature based on environmental heat stress indices.

\section{Discussion}

The aim of this study was to develop a series of empirical equations for the prediction of PWC in the heat. Using data for an average group of young males with heterogeneous fitness and body characteristics, we produced a series of equations where PWC can be predicted based on a series of heat stress assessment metrics, any combination of air temperature and relative humidity, or mean skin temperature. The workload employed is relevant to occupational scenarios, which require moderate to heavy physical work, such that we do not encourage the use of our findings for applications outside of this domain, i.e. maximal athletic performance. All equations took a nonlinear, sigmoidal form (Eqs. 8 and 9), which implies a flattening of PWC loss at the extreme heat levels (i.e. WBGT $>40^{\circ} \mathrm{C}$ ). No field data exist that examines PWC at such extreme heat levels, and in line with previous suggestions, work output in these environments is more due to the heat capacity of the body, compared with the change in environmental heat load (Bröde et al. 2017; Kjellstrom et al. 2018); i.e. even in the most extreme conditions, a brief period 
Table 3 Equations linking physical work capacity to the thermal climate

\begin{tabular}{|c|c|c|c|c|c|}
\hline $\begin{array}{l}\text { Heat stress } \\
\text { metric }\end{array}$ & Range & Equation: $\mathrm{PWC}=$ & $R^{2}$ & RMSE & Index accounts for solar radiation? \\
\hline$T_{\mathrm{a}}$ and humidity & $\begin{array}{l}\frac{T_{\mathrm{a}}}{15}-50^{\circ} \mathrm{C} \\
\frac{\mathrm{RH}}{20-80 \%}\end{array}$ & $\frac{100}{1+\left[\frac{(-12.28 L n(\mathrm{RH})+87.99}{T_{\mathrm{a}}}\right]^{[-2.21 L n(\mathrm{RH})+2.63]}}$ & .98 & 3.09 & No \\
\hline Heat index & $14-85^{\circ} \mathrm{C}$ & $\frac{100}{1+\left(\frac{55.47}{\text { heat index }}\right)^{-2.90}}$ & .97 & 4.10 & No \\
\hline Humidex & $13-71^{\circ} \mathrm{C}$ & $\frac{100}{1+\left(\frac{54.50}{\text { humidex }}\right)^{-4.10}}$ & .96 & 4.86 & No \\
\hline$T_{\mathrm{wb}}$ & $10-39^{\circ} \mathrm{C}$ & $\frac{100}{1+\left(\frac{30.98}{T_{\mathrm{wb}}}\right)^{-5.90}}$ & .95 & 5.60 & No \\
\hline UTCI & $15-63^{\circ} \mathrm{C}$ & $\frac{100}{1+\left(\frac{45.33}{\text { UTCI }}\right)^{-4.30}}$ & .94 & 5.90 & Yes \\
\hline WBGT & $12-40^{\circ} \mathrm{C}$ & $\frac{100}{1+\left(\frac{33.63}{\text { WBGT }}\right)^{-6.33}}$ & .94 & 5.94 & Yes \\
\hline
\end{tabular}

WBGT wet bulb globe temperature, $T_{w b}$ aspirated wet bulb temperature, UTCI universal thermal climate index, $T_{a}$ air temperature, RMSE root-meansquare error, $L n$ natural logarithm

of work is possible, assuming the worker can move to a cooler climate after the work is finished.

Figure 2 shows PWC based on several environmental assessment metrics. While Table 3 shows that the heat index formed models with the least residual variance, the WBGT and UTCI are able to account for solar radiation, so these models can be used for indoor and outdoor working scenarios. Our ongoing work aims to form correction factors so that indices that do not account for solar radiation can also be used to predict PWC outdoors (Foster et al. 2020b). A pooled model incorporating both clothing conditions is recommended for predicting PWC on a global scale, since it represents a generalised approximation that does not account for the subtle differences elicited by the clothing. The difference in parameter ( $P W C 50$ and Hillslope) outcomes between conditions of high- and low-clothing coverage was consistent but minimal (Fig. 4). This is explained by the use of a reference condition in which the same clothing was worn, rather than comparing all to a minimally clothed condition (Fig. 5).

PWC as a function of air temperature and relative humidity is displayed as a contour plot in Fig. 3. Here, we show that $T_{\mathrm{a}}$ alone is a relatively poor predictor of PWC, which has implications for models that do not consider humidity in addition to temperature (Hsiang et al. 2017; Zivin and Neidell 2014). Regarding the

Table 4 Equations for prediction of physical work capacity, thermal sensation, and thermal comfort due to changes in skin temperature during physical work

\begin{tabular}{llll}
\hline Outcome & Equation & $R^{2}$ & RMSE \\
\hline Physical work capacity \% & \multicolumn{1}{c}{100} & .88 & 8.42 \\
& $1+\left[\frac{36.06}{T_{\text {skin }}}\right]^{-26.57}$ & & \\
Thermal sensation & $0.13 \cdot e^{0.15 T_{\text {skin }}}$ & .84 & 2.77 \\
Thermal comfort & $0.47 T_{\text {skin }}-13.06$ & .73 & 0.44 \\
\hline
\end{tabular}

$T_{\text {skin }}$ skin temperature in ${ }^{\circ} \mathrm{C}, R M S E$ root-mean-square error. Equations only valid for skin temperatures between 30 and $38^{\circ} \mathrm{C}$ clothing effect, it is shown in Fig. 4 that although the PWC50 parameter (the value of $x$ at 50\% PWC) is similar between conditions, the Hillslope coefficient is smaller in the clothed condition, resulting in a shallower curve. The latter indicates a protective effect of high-clothing coverage in high temperatures (ambient temperature $>$ skin temperature), but also a negative impact in milder conditions (skin temperature $>$ ambient temperature). This notion is not surprising from a biophysical point of view and is supported by research examining the impact of clothing coverage in heat stressed humans (McLellan and Havenith 2016).

\section{Skin temperature predicts PWC and perceptual responses to heat stress}

We additionally modelled the thermometric and perceptual responses across the full span of environmental conditions tested. At a fixed heart rate, which is a surrogate for self-paced physical workloads, Fig. 5a-c demonstrates that rising skin temperature mediates heat-induced reductions in PWC, whereas internal body temperature and mean body temperature (a weighted combination of skin temperature and core temperature) are not useful predictors. Although rising core temperature is the primary risk factor for heat stroke (Leon and Bouchama 2015), this event is rare in most occupations, especially if workers can self-pace (Miller et al. 2011). Rises in core temperature are primarily driven by metabolic workload (Cramer and Jay 2015), and since workload decreases in hot conditions due to self-pacing (Miller et al. 2011), core temperatures rarely reach $39^{\circ} \mathrm{C}$ in the field (Kalkowsky and Kampmann 2006; Miller et al. 2011) and in our dataset. In contrast, rising skin temperature is largely driven by the environment, where hot skin increases workers' heart rate and perception of effort. We criticised the approach of Dunne et al. (2013), using the ACGIH work/rest recommendations to predict physical productivity, because the guidelines are designed to prevent core temperature exceeding $38^{\circ} \mathrm{C}$ and are thus highly 
Fig. 3 The change in physical work capacity percent as a function of air temperature and relative humidity. A graphical representation of the formula in Table 3 indicates the importance of humidity for the prediction of work capacity at a given air temperature. Values within the matrix indicate total physical work capacity as a percentage. Values are extrapolated where physical work capacity is $<25 \%$

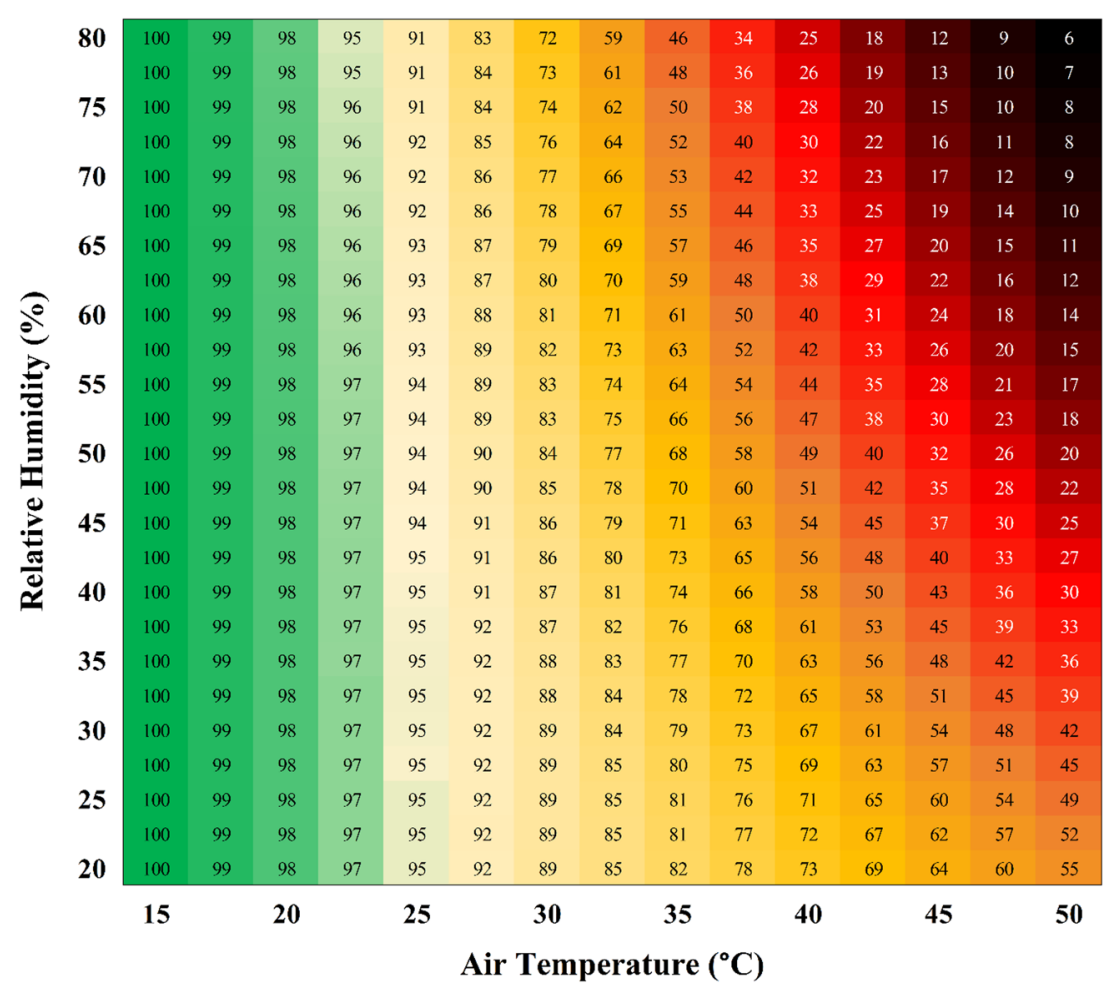

conservative. Although our data indicate modest rises in core temperature, we emphasise that those values (as in Fig. 5c) are the average of a work bout, not the maximum. The core-to-skin temperature gradient equally shows good predictive value; however, further analysis showed that this is almost completely based on the skin temperature change.
The rating of perceived exertion (RPE) scale measures the participants' subjective assessment of their physical effort (Borg 1982). The psychophysiological basis for the scale implies that RPE is strongly linked to cardiovascular strain, captured by heart rate, and thus, using the constant heart rate paradigm, RPE was expected to be constant. Figure 5 f confirms that RPE did not

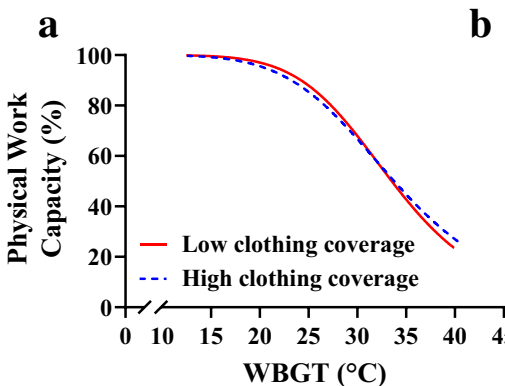

b

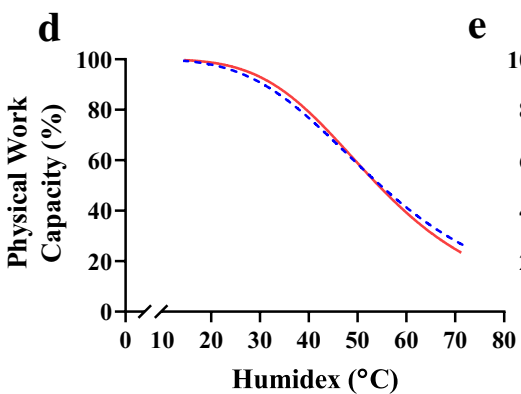

e

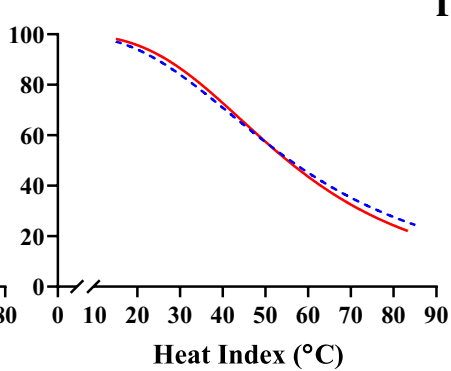

c

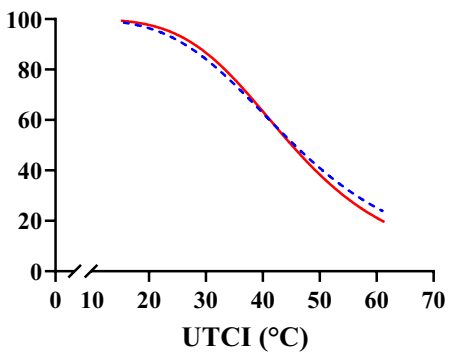

f

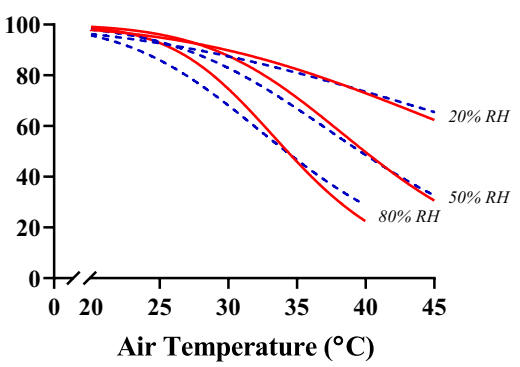

Fig. 4 Clothing-specific models for physical work capacity. Models are presented based on low (red lines) or high (blue lines) levels of clothing coverage. Unlike the pooled data shown previously, the models here can be used for specific industries based on whether protective clothing is required. High-clothing coverage was detrimental in mild heat stress but offered some protective effect at more extreme heat. The thermal properties of each ensemble are described in the methods. Model analytics are available as supplementary material 
a

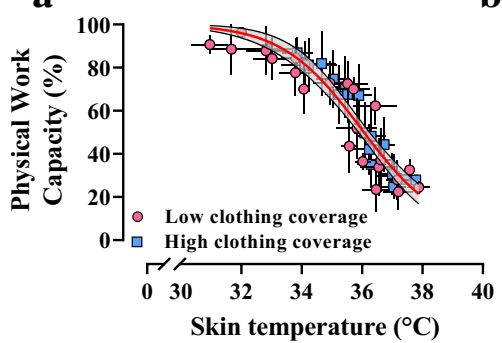

d

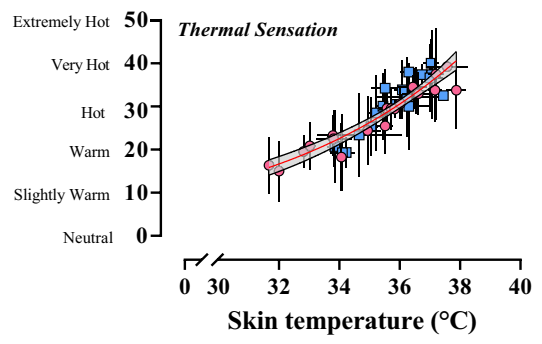

Fig. 5 Performance and perceptual responses to heat are mediated by increased skin temperature. The change in physical work capacity (PWC) with heat (top panel) is predicted well by average skin temperature over the exposure (a), but not the average core (b) or the average mean body temperature (c). The data used to form projections are taken as the average physical work capacity from each air temperature and humidity

change as a function of heat stress intensity, i.e. remains linked to heart rate in the heat, despite increases in thermal perception in hot climates (Fig. 5d and e). These results support recent commentary (Lloyd and Havenith 2019) and imply that reducing skin temperature should be the primary intervention to maintaining PWC and thermal perception in hot workplaces. Equations linking PWC to skin temperature, thermal sensation, and thermal comfort are displayed in Table 4.

\section{Comparison with previous models}

It is useful to compare our model against WBGT-based models described in the introduction of our paper (Dunne et al. 2013; Kjellstrom et al. 2018). As shown in Fig. 6, a comparison is only available for WBGT since previous models were developed for this index only. Due to the incorporation of a cool reference condition and the high sensitivity of heart rate to changes in heat stress, our model better detects reductions in PWC in mild heat, compared to models developed by Dunne et al. (2013) and Kjellstrom et al. (2018), which only predict PWC reductions above $25^{\circ} \mathrm{C}$ WBGT. The high sensitivity of our model allows for a more accurate quantification of PWC with the more subtle climatic alterations observed in temperate climates, rather than the exclusive consideration of more severe conditions. Importantly, it is well established that $25^{\circ} \mathrm{C}$ WBGT already far exceeds optimal ambient conditions for human physical performance (Ely et al. 2007; Galloway and Maughan 1997; Taylor et al. 1963). In contrast, our reference condition $\left(15^{\circ} \mathrm{C} ; 12^{\circ} \mathrm{C}\right.$ c
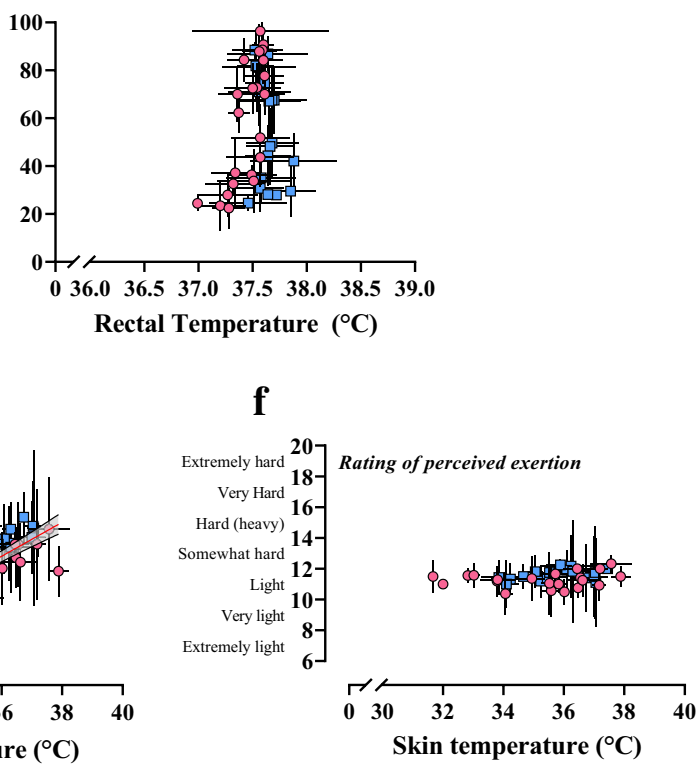

combination, pooling that of low- (pink circles) and high- (blue squares) clothing coverage trials. Perceptual responses to heat can be predicted by skin temperature (d and e). There was no change in perceived exertion (f) with climatic stress, supporting the notion that perceived effort is primarily mediated by heart rate

WBGT) represents a more optimal environment for human physical work output (Taylor et al. 1963), allowing us to document PWC reductions in environments as mild as $18^{\circ} \mathrm{C}$ WBGT.

Although our model is more sensitive to low heat stress compared to both previous models, we also observed a shallower decline in PWC when WBGT surpassed $25^{\circ} \mathrm{C}$ compared with Dunne's (2013) and Kjellstrom et al.'s (2018) models. PWC estimations from our model and that of Kjellstrom et al. (2018) produce similar values around $30-5^{\circ} \mathrm{C}$ WBGT, where the lines cross over. Due to Kjellstrom et al.'s choice to select $10 \%$ PWC as a lower limit, and the lack of data above $33^{\circ} \mathrm{C}$ WBGT, their predicted PWC values drop substantially below our data in this area. Our lowest PWC values observed were around $25-30 \%$ at $40^{\circ} \mathrm{C}$ WBGT and the curve suggests further drops above this level of heat stress. Such extreme WBGT values are very rarely encountered, but since workplace WBGT levels up to $35^{\circ} \mathrm{C}$ have been reported in various industries in India (Venugopal et al. 2015), workplace WBGTs $\geq 40^{\circ} \mathrm{C}$ are possible with future global warming.

\section{Limitations}

There are several potential limitations of the current study that should be considered. The first is the use of mostly British participants, assumed to be unacclimatised to heat due to infrequent hot weather and minimal core temperature increases with our protocol, which are normally required for physiological adaptation (Fox et al. 1963). However, aerobic fitness is a 


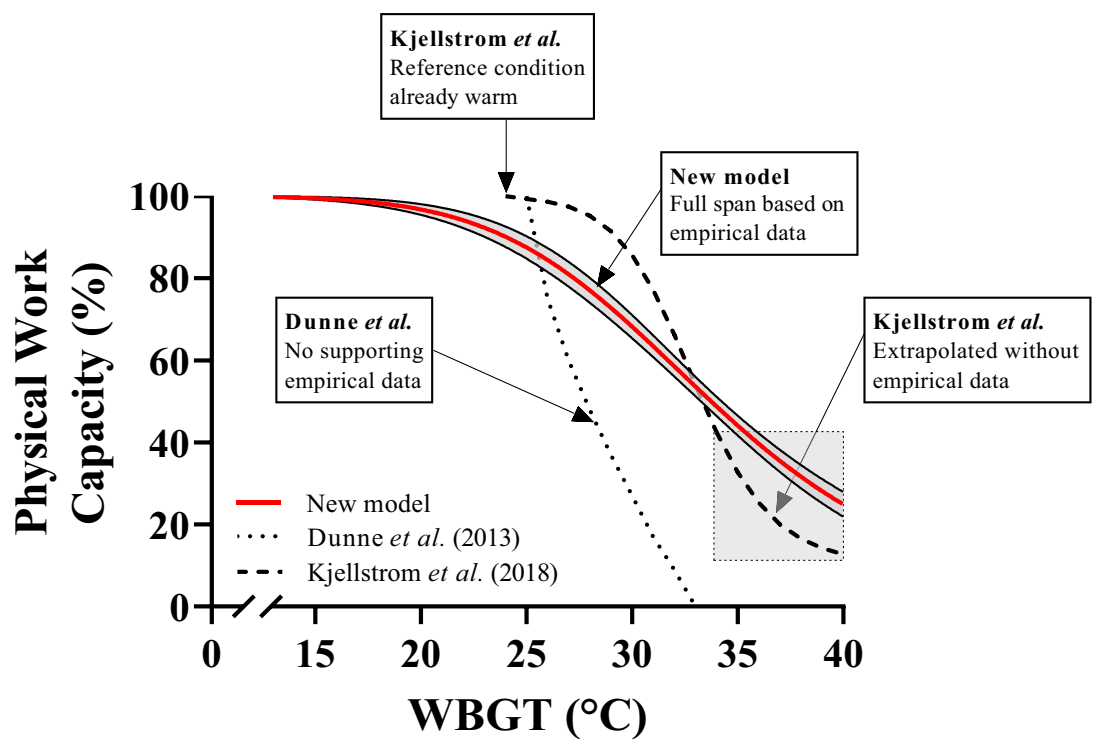

Fig. 6 The relationship between PWC and WBGT for past models and the new model. Deriving relations based on work/rest ratios (dotted line, Dunne et al. (2013)) or from past field data (dashed line, Kjellstrom et al. 2018) underestimate the effect of moderate heat stress and overestimate the effects of extreme heat stress, resulting in unrealistically steep declines in PWC. The red line shows the new model, based on Fig. 2a, where we

major parameter that governs PWC in hot climates (Foster et al. 2020a), and thermoregulatory modelling indicates that those in a high state of acclimatisation (with normal fitness) exhibit a similar heat stress response to those of high fitness (Havenith 2001). The inclusion of participants with high fitness levels therefore does reflect part of the impact acclimatisation would have on the data. Although our model does not account for those who are simultaneously highly fit and acclimatised, these individuals are rare and account for $1-5 \%$ of the population average (Kaminsky et al. 2015).Second, only relatively young, healthy, adult males were recruited, raising the question about the older population and female workers. There is ample evidence however that both older and female workers, when healthy, respond similar to heat exposures as their younger counterparts, as long as their fitness levels and, to a lesser degree, their anthropometrics are considered (Cramer and Jay 2015; Havenith et al. 1995; Havenith and van Middendorp 1990; Notley et al. 2019). Hence, by incorporating a wide range of individual levels of fitness, the results of the present study should be representative of the general population, apart from very low fitness individuals, or those with underlying health conditions, who may respond differently. Thus, when translating to future impact assessment of global warming, our data represents the best-case scenario.

A third consideration is the choice for the type of work performed. Ideally, a range of work intensities and types of work would be studied, including lower working heart rates, upper body (manual work with hands arms mainly) only work, and combined upper and lower body work. Unfortunately, a study of the full range of specific activities in industry and their interaction with climate, while relevant, is collected data up to $40^{\circ} \mathrm{C}$ WBGT. The new model is more sensitive to mild/moderate heat compared with previous work. The grey box indicates the area in which the Kjellstrom et al. (Kjellstrom et al. 2018) model is extrapolated beyond empirical data and an overestimation of the climate effect is present

not feasible, given the plethora of possible activities. In the paradigm used here, the work is performed mainly as lower body work. The main purpose of the work is to create the metabolic load as well as generate the associated heat in the body. In terms of the ratio of heat produced to metabolic rate, this would not be dissimilar for other work types. Further, the work level used does not induce excessive muscle fatigue in the tests; thus, different fatigue speeds for different work types would not be expected to have affected the outcomes.

A fourth consideration is that the equations presented are, at present, only valid for relatively low wind conditions and without solar radiation. Follow-up work from our group shows that the UTCI scale is the optimal climatic index when accounting for higher wind speeds and solar radiation (Foster et al. 2020b; Smallcombe et al. 2019b). The WBGT predicted PWC well during increased solar radiation, but does not account for the dynamic impact of wind; i.e. high wind can increase heat strain in hot dry environments (Morris et al. 2019; Smallcombe et al. 2019b). A fifth consideration is that the models presented in this paper (and their analytics) are developed from group means, and the reported accuracy is not representative of all individuals. Given the wide variation in fitness levels and body characteristics, our models represent a typical group of workers engaged in physical labour. Despite that, in our follow-up work, we modelled the impact of individual fitness on the PWC curves, explaining a large portion of the individual variation (Foster et al. 2021). It should also be noted that we considered using lower heart rates. Some sources suggest an average maximal heart rate over the full working day (rest+work periods average) of 110 b. $\min ^{-1}$. When piloting this, we found already in the mid-range 
of heat stress that participants reached this at rest, so without activity. Thus, this was not a workable model. In real work, for such low work rates, workers would accept an increase of heart rate by heat exposure to around $130 \mathrm{~b} \cdot \mathrm{min}^{-1}$ as discussed, i.e. using their cardiovascular reserve. Thus, for very low workloads, the present model may be on the conservative side, as it does not include this reserve of increasing the HR to 130 from a lower work baseline.

A final potential limitation is the use of a 1-h work simulation, and not a full day work simulation. Our group is actively investigating the extent to which our model predicts PWC across a full working day, with preliminary results implying limited impact of work duration (1 versus 6 hourly work cycles in a day) on PWC until conditions are extreme (WBGT $>35^{\circ} \mathrm{C}$ ) (Smallcombe et al. 2019a). It appears that our model predicts full day PWC within 5\% for most relevant climates encountered on Earth and with existing climate conditions if water is available for rehydration ad libitum during rest periods.

\section{Conclusions}

In summary, we provide new empirical models for changes to physical work capacity (PWC) under a wide variety of environmental conditions, which are based on a range of commonly used climate indices that can be used on the macro level to estimate the impact of heat on productivity and the cost of future climate change for physical work under different $\mathrm{CO}_{2}$ emission scenarios. In conjunction with weather forecasting, the model can also be used on the micro level to estimate dayto-day production losses within a given industry across the local climate range. To our knowledge, this is the first study to provide empirical estimations for PWC based on a large dataset over a wide range of climatic conditions using a wide variety of different heat indices, broadening the scope for future application.

Supplementary Information The online version contains supplementary material available at https://doi.org/10.1007/s00484-021-02105-0.

Funding Funding was provided by 'HEAT-SHIELD', European Union's Horizon 2020 research and innovation programme under grant agreement no. 668786 .

Open Access This article is licensed under a Creative Commons Attribution 4.0 International License, which permits use, sharing, adaptation, distribution and reproduction in any medium or format, as long as you give appropriate credit to the original author(s) and the source, provide a link to the Creative Commons licence, and indicate if changes were made. The images or other third party material in this article are included in the article's Creative Commons licence, unless indicated otherwise in a credit line to the material. If material is not included in the article's Creative Commons licence and your intended use is not permitted by statutory regulation or exceeds the permitted use, you will need to obtain permission directly from the copyright holder. To view a copy of this licence, visit http://creativecommons.org/licenses/by/4.0/.

\section{References}

American College of Sports Medicine (2013) Lippincott Williams \& Wilkins 9781609136055

Andersen KL (1978) Habitual physical activity and health. 188

Armstrong LE, Maresh CM, Castellani JW, Bergeron MF, Kenefick RW, LaGasse KE et al (1994) Urinary indices of hydration status. Int J Sport Nutr 4:265-279. https://doi.org/10.1123/ijsn.4.3.265

Bain AR, Jay O (2011) Does summer in a humid continental climate elicit an acclimatization of human thermoregulatory responses? Eur $\mathrm{J}$ Appl Physiol 111:1197-1205. https://doi.org/10.1007/s00421-0101743-9

Bernard TE, Kenney WL (1994) Rationale for a personal monitor for heat strain. Am Ind Hyg Assoc J 55:505-514. https://doi.org/10.1080/ 15428119491018772

Bernard TE, Pourmoghani M (1999) Prediction of workplace wet bulb global temperature. Appl Occup Environ Hyg 14:126-134. https:// doi.org/10.1080/104732299303296

Borg GA (1982) Psychophysical bases of perceived exertion. Med Sci Sports Exerc 14:377-381

Bröde P, Fiala D, Błażejczyk K, Holmér I, Jendritzky G, Kampmann B et al (2012) Deriving the operational procedure for the universal thermal climate index (UTCI). Int J Biometeorol 56:481-494 https://doi.org/10.1007/s00484-011-0454-1

Bröde P, Fiala D, Lemke B, Kjellstrom T (2017) Estimated work ability in warm outdoor environments depends on the chosen heat stress assessment metric. Int J Biometeorol:1-15 https://doi.org/10.1007/ s00484-017-1346-9

Chen WY, Elizondo RS (1974) Peripheral modification of thermoregulatory function during heat acclimation. J Appl Physiol 37:367-373

Chou TH, Akins JD, Crawford CK, Allen JR, Coyle EF (2019) Low stroke volume during exercise with hot skin is due to elevated heart rate. Med Sci Sports Exerc 51:2025-2032 https://doi.org/10.1249/ MSS.0000000000002029

Cramer MN, Jay O (2015) Explained variance in the thermoregulatory responses to exercise: the independent roles of biophysical and fitness/fatness-related factors. J Appl Physiol 119:982-989 https:// doi.org/10.1152/japplphysiol.00281.2015

Cramer MN, Jay O (2019) Partitional calorimetry. J Appl Physiol 126: 267-277. https://doi.org/10.1152/japplphysiol.00191.2018

Dunne J, Stouffer R, John J (2013) Reductions in labour capacity from heat stress under climate warming. Nat Clim Chang 3:563-566. https://doi.org/10.1038/nclimate1827

Ely MR, Cheuvront SN, Roberts WO, Montain SJ (2007) Impact of weather on marathon-running performance. Med Sci Sports Exerc 39:487-493. https://doi.org/10.1249/mss.0b013e31802d3aba

Flouris AD, Dinas PC, Ioannou LG, Nybo L, Havenith G, Kenny GP et al (2018) Workers' health and productivity under occupational heat strain: a systematic review and meta-analysis. Lancet Planet Heal 2:e521-e531. https://doi.org/10.1016/S2542-5196(18)30237-7

Foster J, Hodder SG, Lloyd AB, Havenith G (2020a) Individual responses to heat stress: implications for hyperthermia and physical work capacity. Front Physiol 11:1147. https://doi.org/10.3389/ fphys.2020.541483

Foster J, Smallcombe J, Hodder S, Jay O, Flouris A, Havenith G (2020b) The impact of solar radiation on human physical work capacity in the heat: interactions between clothing, air temperature, and humidity. in Virtual 8th International Conference on the Physiology and 
Pharmacology of Temperature Regulation (vPPTR) (Vancouver, Canada)

Foster J, Smallcombe J, Hodder S, Jay O, Flouris A, Morris N et al (2021) [submitted paper] Aerobic fitness as a parameter of importance for labour loss in the heat. J Sci Med Sport

Fox RH, Goldsmith R, Kidd DJ, Lewis HE (1963) Blood flow and other thermoregulatory changes with acclimatization to heat. J Physiol 166:548-562

Galloway SD, Maughan RJ (1997) Effects of ambient temperature on the capacity to perform prolonged cycle exercise in man. Med Sci Sports Exerc 29:1240-1249

Garrett AT, Rehrer NJ, Patterson MJ (2011) Induction and decay of shortterm heat acclimation in moderately and highly trained athletes. Sports Med 41:757-771. https://doi.org/10.2165/11587320000000000-00000

Hamermesh DS, Frazis H, Stewart J (2005) Data watch: the American time use survey. J Econ Perspect 19:221-232. https://doi.org/10. $1257 / 0895330053148029$

Havenith G (2001) Individualized model of human thermoregulation for the simulation of heat stress response. J Appl Physiol 90:1943-1954

Havenith G, Fiala D (2015) Thermal indices and thermophysiological modeling for heat stress. Compr Physiol 6:255-302. https://doi. org/10.1002/cphy.c140051

Havenith G, van Middendorp H (1990) The relative influence of physical fitness, acclimatization state, anthropometric measures and gender on individual reactions to heat stress. Eur J Appl Physiol Occup Physiol 61:419-427. https://doi.org/10.1007/BF00236062

Havenith G, Inoue Y, Luttikholt V, Kenney WL (1995) Age predicts cardiovascular, but not thermoregulatory, responses to humid heat stress. Eur J Appl Physiol Occup Physiol 70:88-96

Hodder SG, Parsons K (2007) The effects of solar radiation on thermal comfort. Int J Biometeorol. https://doi.org/10.1007/s00484-0060050-y

Hsiang S, Kopp R, Jina A, Rising J, Delgado M, Mohan S et al (2017) Estimating economic damage from climate change in the United States. Science (80- ) 356:1362-1369. https://doi.org/10.1126/ science.aal4369

Hübler M, Klepper G, Peterson S (2008) Costs of climate change: the effects of rising temperatures on health and productivity in Germany. Ecol Econ 68:381-393. https://doi.org/10.1016/j. ecolecon.2008.04.010

Ioannou LG, Tsoutsoubi L, Samoutis G, Bogataj LK, Kenny GP, Nybo L et al (2017) Time-motion analysis as a novel approach for evaluating the impact of environmental heat exposure on labor loss in agriculture workers. Temperature. https://doi.org/10.1080/23328940.2017. 1338210

ISO10551 (2001) Ergonomics of the thermal environment — assessment of the influence of the thermal environment using subjective judgement scales

ISO9920 (2009) International Standardisation Organisation. Ergonomics of the thermal environment - estimation of thermal insulation and water vapour resistance of a clothing ensemble

Jay O, Hoelzl R, Weets J, Morris N, English T, Nybo L et al (2019) Fanning as an alternative to air conditioning - a sustainable solution for reducing indoor occupational heat stress. Energy Build 193:9298. https://doi.org/10.1016/j.enbuild.2019.03.037

Kalkowsky B, Kampmann B (2006) Physiological strain of miners at hot working places in German coal mines. Ind Health 44:465-473

Kaminsky LA, Arena R, Myers J (2015) Reference standards for cardiorespiratory fitness measured with cardiopulmonary exercise testing. Mayo Clin Proc 90:1515-1523. https://doi.org/10.1016/j.mayocp. 2015.07.026

Kjellstrom T, Freyberg C, Lemke B, Otto M, Briggs D (2018) Estimating population heat exposure and impacts on working people in conjunction with climate change. Int J Biometeorol 62:291-306. https:// doi.org/10.1007/s00484-017-1407-0
Leon LR, Bouchama A (2015) Heat stroke. Compr Physiol 5:611-647. https://doi.org/10.1002/cphy.c140017

Lloyd A, Havenith G (2019) Comment on: subjective thermal strain impairs endurance performance in a temperate environment. Physiol Behav 204:275-276. https://doi.org/10.1016/j.physbeh. 2019.03.006

Ludlow LW, Weyand PG (2017) Walking economy is predictably determined by speed, grade, and gravitational load. J Appl Physiol 123: 1288-1302. https://doi.org/10.1152/japplphysiol.00504.2017

Mairiaux P, Malchaire J (1985) Workers self-pacing in hot conditions: a case study. Appl Ergon 16:85-90. https://doi.org/10.1016/00036870(85)90209-1

Malchaire J, Piette A, Kampmann B, Mehnert P, Gebhardt H, Havenith G et al (2001) Development and validation of the predicted heat strain model. Ann Occup Hyg 45:123-135. https://doi.org/10.1016/ S0003-4878(00)00030-2

Marino FE, Mbambo Z, Kortekaas E, Wilson G, Lambert MI, Noakes TD et al (2000) Advantages of smaller body mass during distance running in warm, humid environments. Pflugers Arch - Eur J Physiol. https://doi.org/10.1007/s004240000432

Masterton J, Richardson F (1979) Humidex: a method of quantifying human discomfort due to excessive heat and humidity. Environment Canada, Atmospheric Environment

Maughan RJ, Otani H, Watson P (2012) Influence of relative humidity on prolonged exercise capacity in a warm environment. Eur J Appl Physiol 112:2313-2321. https://doi.org/10.1007/s00421-011-22067

McLellan TM, Havenith G (2016) Protective clothing ensembles and physical employment standards. Appl Physiol Nutr Metab. https:// doi.org/10.1139/apnm-2015-0474

Miller V, Bates G, Schneider JD, Thomsen J (2011) Self-pacing as a protective mechanism against the effects of heat stress. Ann Occup Hyg 55:548-555. https://doi.org/10.1093/annhyg/mer012

Morris NB, English T, Hospers L, Capon A, Jay O (2019) The effects of electric fan use under differing resting heat index conditions: a clinical trial. Ann Intern Med. https://doi.org/10.7326/M19-0512

Notley SR, Lamarche DT, Meade RD, Flouris AD, Kenny GP (2019) Revisiting the influence of individual factors on heat exchange during exercise in dry heat using direct calorimetry. Exp Physiol 104: 1038-1050. https://doi.org/10.1113/EP087666

Otani H, Kaya M, Tamaki A, Watson P, Maughan RJ (2016) Effects of solar radiation on endurance exercise capacity in a hot environment. Eur J Appl Physiol 116:769-779. https://doi.org/10.1007/s00421016-3335-9

Pal JS, Eltahir EAB (2016) Future temperature in southwest Asia projected to exceed a threshold for human adaptability. Nat Clim Chang 6:197-200. https://doi.org/10.1038/nclimate2833

Pandolf KB, Givoni B, Goldman RF (1977) Predicting energy expenditure with loads while standing or walking very slowly. J Appl Physiol Respir Environ Exerc Physiol 43:577-581. https://doi.org/ 10.1152/jappl.1977.43.4.577

Parsons KC (2010) Human thermal environments, 2nd edn. Taylor \& Francis, London. https://doi.org/10.4324/9780203302620 chapter_1

Ramanathan NL (1964) A new weighting system for mean surface temperature of the human body. J Appl Physiol 19:531-533. https://doi. org/10.1152/jappl.1964.19.3.531

Rana R, Kusy B, Jurdak R, Wall J, Hu W (2013) Feasibility analysis of using humidex as an indoor thermal comfort predictor. Energy Build 64:17-25. https://doi.org/10.1016/j.enbuild.2013.04.019

Raymond C, Matthews T, Horton RM (2020) The emergence of heat and humidity too severe for human tolerance. Sci Adv 6:eaaw1838. https://doi.org/10.1126/sciadv.aaw1838

Rothfusz LP (1990) The heat index "equation" (or, more than you ever wanted to know about heat index) 
Rowell LB (1974) Human cardiovascular adjustments to exercise and thermal-stress. Physiol Rev 54:75-159

Sahu S, Sett M, Kjellstrom T (2013) Heat exposure, cardiovascular stress and work productivity in rice harvesters in India: implications for a climate change future. Ind Health 51:424-431

Smallcombe J, Foster J, Hodder S, Jay O, Flouris AD, Havenith G (2019a) Quantifying physical work capacity in the heat: one hour vs full day exposure. in International Conference on Environmental Ergonomics, 128

Smallcombe J, Foster J, Hodder S, Jay O, Flouris A, Griggs K et al (2019b) Impact of fan use on physical work capacity in extreme heat. Med Sci Sports Exerc 51:15. https://doi.org/10.1249/01.mss. 0000560531.57940.a8

Taylor HL, Wang Y, Rowell L, Blomqvist G (1963) The standardization and interpretation of submaximal and maximal tests of working capacity. Pediatrics 32

Venugopal V, Chinnadurai JS, Lucas RAI, Kjellstrom T (2015) Occupational heat stress profiles in selected workplaces in India.
Int J Environ Res Public Health 13. https://doi.org/10.3390/ ijerph13010089

Vogt JJ, Libert JP, Candas V, Daull F, Mairjaux P (1983) Heart rate and spontaneous work-rest cycles during exposure to heat. Ergonomics 26:1173-1185. https://doi.org/10.1080/00140138308963453

Waterhouse J, Edwards B, Bedford P, Hughes A, Robinson K, Nevill A et al (2004) Thermoregulation during mild exercise at different circadian times. Chronobiol Int 21:253-275. https://doi.org/10.1081/ CBI-120037799

Wyndham CH (1969) Adaptation to heat and cold. Environ Res 2:442469. https://doi.org/10.1016/0013-9351(69)90015-2

Wyndham CH (1973) The effects of heat stress upon human productivity. Arch Sci Physiol (Paris) 27:491-497

Zander K, Botzen W, Oppermann E (2015) Heat stress causes substantial labour productivity loss in Australia. Nat Clim 5:647-651

Zivin JG, Neidell M (2014) Temperature and the allocation of time: implications for climate change. J Labor Econ 32:1-26. https://doi. org/10.1086/671766 